\title{
Análisis de la respuesta mecánica de recubrimientos elaborados mediante proyección térmica por plasma usando la medición de esfuerzos residuales y el método de elementos finitos a escala estructural: efecto de la red de poros
}

\author{
Mechanical responses of plasma sprayed coatings from residual stress \\ measurements and finite element analysis: effect of the pore network architecture
}

Claudia Constanza Palacio Espinosa ${ }^{1}$

Recibido 3 de junio de 2011, aceptado 17 de octubre de 2011

Received: June 3, $2011 \quad$ Accepted: October 17, 2011

\begin{abstract}
RESUMEN
En el presente trabajo se estudia la respuesta mecánica de recubrimientos elaborados mediante proyección térmica por plasma ante la presencia de esfuerzos residuales y campos de esfuerzos generados por deformaciones. Para lograr este propósito, se midieron los esfuerzos residuales durante el proceso de elaboración de recubrimientos de $\mathrm{TiO}_{2}-\mathrm{Cu}$ y se evaluó el efecto de la red de poros en la estructura de la capa obtenida a partir de imágenes de alta resolución de Microscopía Electrónica de Barrido (MEB) obtenidas de los recubrimientos de $\mathrm{Al}_{2} \mathrm{O}_{3}-13 \mathrm{TiO}_{2}$, las cuales fueron posteriormente procesadas y discretizadas para su análisis mediante el Método de Elementos Finitos (MEF). Algunos parámetros operacionales empleados para elaborar los recubrimientos, tales como el flujo de gases y la corriente eléctrica, necesarios para formar el plasma, mostraron tener influencia en el contenido de poros, densidad de grietas y orientación de las mismas y en la magnitud de los esfuerzos residuales provocados. Los esfuerzos residuales determinados a partir de mediciones de deflexión durante la elaboración de los recubrimientos de $\mathrm{TiO}_{2}-\mathrm{Cu}$, permitieron identificar su naturaleza, mientras que las simulaciones numéricas mostraron la gran influencia de la red de poros sobre el campo de esfuerzos de los recubrimientos de $\mathrm{Al}_{2} \mathrm{O}_{3}-13 \mathrm{TiO}_{2}$. Del mismo modo, mediante MEF se evaluaron otras composiciones teóricas a través de la modificación de las propiedades mecánicas intrínsecas de las fases constituyentes. Para los casos considerados, las simulaciones mostraron la pobre influencia que tiene la presencia de los constituyentes secundarios en el campo de esfuerzos de los recubrimientos.
\end{abstract}

Palabras clave: Recubrimientos por plasma, propiedades mecánicas, deflexión, elementos finitos, microestructura.

\section{ABSTRACT}

This study aims a better understanding residual stress and stress field within composite plasma sprayed coatings submitted to strains. In order to reach out this objective, residual stress techniques can be implemented for measurements during elaboration of coatings and finite element analyses (FEA) can be conducted on domains resulting from the discretization of Scanning Electron Microscopy (SEM) high resolution pictures of coating structures. $\mathrm{TiO}_{2}-\mathrm{Cu}$ and $\mathrm{Al}_{2} \mathrm{O}_{3}-13 \mathrm{TiO} \mathrm{O}_{2}$ plasma sprayed coatings were considered for demonstrative purposes regarding residual stress measurements and effect of the pore network architecture, respectively. Several operating parameters permitted to manufacture coatings exhibiting different magnitude of residual stress and several porous architectures, in terms of pore content, crack density and crack orientation. Residual stress determined from measurements of deflection during elaboration of $\mathrm{TiO}_{2}$-Cu coatings allow to identify their nature while numerical simulations show the very significant influence of the network architecture on the stress field within the $\mathrm{Al}_{2} \mathrm{O}_{3}-13 \mathrm{TiO}_{2}$ coatings. Based on the same analyzed structures, other theoretical compositions were considered in the finite

\footnotetext{
1 Facultad de Ciencias y Humanidades. Universidad EAFIT. 050022 Carrera 49 N 7 Sur - 50. Medellín, Colombia. E-mail: cpalac12@eafit.edu.co
} 
element analyses by modifying the intrinsic mechanical properties of the phases. For the considered cases, simulations show the poor influence of the nature of the secondary phase on the stress field within the coatings. Beside these results, the paper proposes a generic methodology and points out its advantages and limits, emphasizing in particular the importance of the representative elementary volume (REV).

Keywords: Plasma coatings, mechanical properties, deflection, finite element analysis, microstructure.

\section{INTRODUCCIÓN}

La integridad de recubrimientos se ve altamente influenciada por la presencia y magnitud de esfuerzos residuales inducidos durante su elaboración. Por lo tanto, conocer su origen facilita su mitigación, ya que permite el control de algunos de los parámetros utilizados en su elaboración.

Adicionalmente, los recubrimientos de materiales compuestos elaborados por proyección térmica poseen propiedades mecánicas aparentes que dependen de las propiedades intrínsecas de cada uno de los constituyentes de la microestructura (matriz, constituyentes secundarios, etc.) conjugadamente con su distribución espacial y características de arquitectura porosa [1-8].

El presente trabajo estudia el efecto de: i) diferentes factores asociados con el proceso de elaboración de recubrimientos mediante proyección térmica por plasma atmosférico; ii) las propiedades intrínsecas de los constituyentes; iii) la arquitectura porosa sobre las propiedades elásticas aparentes (módulo de Young) de recubrimientos de $\mathrm{Al}_{2} \mathrm{O}_{3}-13 \mathrm{TiO}_{2}$; y iv) los parámetros de proyección sobre la aparición de esfuerzos residuales en recubrimientos de $\mathrm{TiO}_{2}-\mathrm{Cu}$. También se pretende ofrecer un mejor entendimiento sobre el comportamiento mecánico de los recubrimientos elaborados por proyección térmica, tanto a nivel microestructural (en escala de unos pocos micrómetros) como macroestructural (en escala de unos pocos cientos de micrómetros) bajo esfuerzos mecánicos simulados por MEF utilizando el software ABAQUS [9], complementado con el software OOF para la discretización del dominio del problema [10] y con el software SCION Image para el tratamiento de las imágenes [11].

\section{PROCEDIMIENTO EXPERIMENTAL}

\section{Preparación de muestras}

Los substratos empleados para la medición de esfuerzos residuales durante la elaboración de los recubrimientos de $\mathrm{TiO}_{2}-\mathrm{Cu}$ consistieron en probetas ALMEN tipo $\mathrm{N}$ grado $1 \mathrm{~S}$ [12], estandarizadas según la norma SAEJ442 [13], fabricadas de lámina de acero al carbono de baja aleación (SAE 1070) y tratadas térmicamente mediante calentamiento hasta austenización a temperatura de $815^{\circ} \mathrm{C}$, seguida de temple y revenido hasta alcanzar durezas en el rango de 72,5-76,0 HRA. La geometría y dimensiones estándar de las probetas ALMEN se presentan en la Figura 1. Estas probetas fueron seleccionadas dada su sensibilidad para registrar pequeñas deformaciones. Los substratos fueron preparados superficialmente con chorro de partículas de corindón de $400 \mu \mathrm{m}$ de diámetro, las cuales fueron proyectadas a una presión de 0,6 MPa utilizando una boquilla de $8 \mathrm{~mm}$ de diámetro interno, ubicada perpendicularmente al substrato y a una distancia de $100 \mathrm{~mm}$. La rugosidad media final $\mathrm{R}_{\mathrm{a}} \operatorname{lograda}$ fue de $3 \mu \mathrm{m}$. Posteriormente los substratos fueron limpiados en un baño ultrasónico de etanol para remover grasa e impurezas antes de la proyección de los polvos.

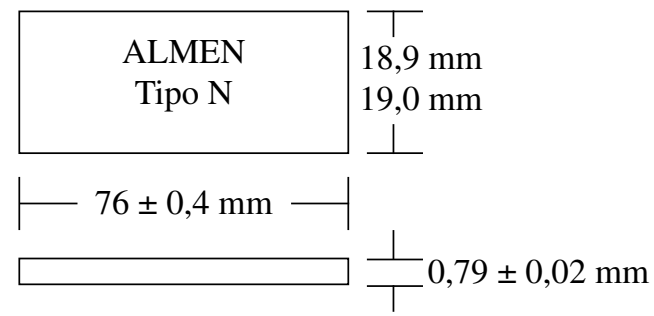

Figura 1. Probetas estándar ALMEN tipo N-1S usadas como substratos para los recubrimientos de rutilo-cobre.

Parámetros operativos de la proyección térmica Se elaboraron recubrimientos de rutilo-cobre $\left(\mathrm{TiO}_{2}-\mathrm{Cu}\right.$, el porcentaje de cobre varió entre $25 \%$ y $33 \%$ en peso) y se emplearon recubrimientos de 
alúmina -óxido de titanio $\left(\mathrm{Al}_{2} \mathrm{O}_{3}-13 \mathrm{TiO}_{2}\right)$ [14]. Todos estos fueron elaborados usando una antorcha de plasma atmosférico tipo F4 (Sulzer-Metco, Wholen, Switzerland), provista de un ánodo de $7 \mathrm{~mm}$ de diámetro (que actúa como boquilla) y empleando diferentes mezclas de $\mathrm{Ar}-\mathrm{H}_{2}$ e intensidades de corriente de arco eléctrico, como se muestra en la Tabla 1 y Tabla 2, respectivamente.

Tabla 1. Parámetros de proyección empleados en la elaboración de los recubrimientos de rutilo-cobre.

\begin{tabular}{|c|c|}
\hline Parámetro & Magnitud \\
\hline Intensidad de corriente de $\operatorname{arco}(\mathrm{A})$ & 500 \\
\hline Flujo másico de $\mathrm{Ar}\left(1 . \mathrm{min}^{-1}\right)$ & 40 \\
\hline Flujo másico de $\mathrm{H}_{2}\left(1 . \mathrm{min}^{-1}\right)$ & 4 \\
\cline { 2 - 2 } & 8 \\
\hline $\begin{array}{c}\text { Flujo de gas de alimentación de } \\
\text { rutilo (l.min }\end{array}$ & 12 \\
\hline $\begin{array}{c}\text { Flujo de gas de alimentación de } \\
\text { cobre (l.min }\end{array}$ & 4,3 \\
\hline $\begin{array}{c}\text { Velocidad de desplazamiento de } \\
\text { las probetas (mm.min }\end{array}$ & 4,5 \\
\hline Distancia de proyección (mm) & 300 \\
\hline Precalentamiento & 125 \\
\hline
\end{tabular}

Los polvos empleados para la fabricación de los recubrimientos de $\mathrm{TiO}_{2}-\mathrm{Cu}$ se obtuvieron de mezclar polvos de Sulzer-Metco Amdry 6505 con polvos Metco 55. Los polvos Amdry 6505 están constituidos por $99,86 \%$ de $\mathrm{TiO}_{2}$ y $0,14 \%$ de otros constituyentes $\left(\mathrm{Al}_{2} \mathrm{O}_{3}, \mathrm{Fe}_{2} \mathrm{O}_{3}, \mathrm{MgO}, \mathrm{SiO}_{2}\right.$ y $\left.\mathrm{CaO}\right)$, sus partículas tienen una geometría irregular y la distribución de tamaño varía entre $\mathrm{d}_{10}=12,5 \mu \mathrm{m}$ y $\mathrm{d}_{90}=42,5 \mu \mathrm{m}$. Por su parte, los polvos Metco 55 poseen $99,2 \%$ de $\mathrm{Cu}$, sus partículas son esféricas y la distribución de tamaño varía entre $\mathrm{d}_{10}=65,5 \mu \mathrm{m}$ $\mathrm{y} \mathrm{d}_{90}=82,4 \mu \mathrm{m}$.

Los polvos de alúmina-óxido de titanio y de cobrerutilo fueron inyectados en el exterior de la antorcha utilizando argón como gas de alimentación y un inyector de $1,8 \mathrm{~mm}$ de diámetro interno. Debido a la marcada diferencia de densidad entre el rutilo y el cobre, estos polvos fueron coinyectados (utilizando simultáneamente un inyector para cada polvo), para evitar su segregación y consecuente distribución irregular en la estructura del recubrimiento obtenido. El flujo de argón utilizado para la inyección de cada uno de estos polvos es mostrado en la Tabla 1. Para la alimentación de los polvos de alúmina-óxido de titanio y de los de rutilo, el inyector fue ubicado a $3 \mathrm{~mm}$ en dirección axial y a $8 \mathrm{~mm}$ en dirección radial, con respecto al centro de la superficie de salida de la boquilla, mientras que para la alimentación de los polvos de cobre (coinyectados con los de rutilo), el inyector fue ubicado a 9 mm en dirección axial. Ver Figura 2. Para la elaboración de los recubrimientos la antorcha realizó ocho pases frente al substrato, empleando relaciones de alimentación de rutilo-cobre de 0-0, 6-2, 6-3 y 10-4 g. $\mathrm{min}^{-1}$ para cada uno de los flujos másicos de $\mathrm{H}_{2}$ presentados en la Tabla 1 .

Durante la elaboración de los recubrimientos obtenidos con los polvos de rutilo-cobre, se realizaron mediciones experimentales de esfuerzos residuales, mientras que con los recubrimientos de alúmina-óxido de titanio se determinaron los módulos de Young aparentes para diferentes condiciones de recubrimiento y nivel de esfuerzo residual. Para ello, las muestras de los recubrimientos de alúmina-rutilo fueron cortadas en sentido paralelo a la dirección de la proyección, montadas en resina époxica y pulidas usando pasta de diamante. El pulido se realizó siguiendo estándares para preparación metalográfica (pulido inicial con papel abrasivo y pulido final con pasta de diamante) en una pulidora semiautomática, para luego ser analizadas mediante Microscopía Electrónica de Barrido (MEB).

\section{Mediciones experimentales realizadas durante la elaboración de recubrimientos de $\mathrm{TiO}_{2}-\mathrm{Cu}$}

La medición de esfuerzos residuales se realizó durante la proyección de los polvos de rutilo y cobre, usando un sistema constituido por la antorcha de plasma, un soporte para las probetas ALMEN, un sistema de enfriamiento, el o los alimentador(es) de polvos y un detector de desplazamientos para registrar la deformación de la probeta, ver Figura 2a. Las probetas se sujetaron en un extremo y se dejaron libres en el extremo opuesto, con el fin de medir la deflexión producida en el extremo libre durante la elaboración del recubrimiento, ver Figura 2b. Los esfuerzos residuales fueron calculados a partir de la ecuación de Stoney, ecuación (1) [15], usando las deflexiones medidas: 
Tabla 2. Parámetros de operación variables para la elaboración de los recubrimientos de alúmina-óxido de titanio [14].

\begin{tabular}{|c|l|c|c|c|}
\hline Item & \multicolumn{1}{|c|}{$\begin{array}{c}\text { Parámetro } \\
\text { variable }\end{array}$} & $\begin{array}{c}\text { Intensidad } \\
\text { corriente } \\
\text { de arco } \\
\text { (A) }\end{array}$ & $\begin{array}{c}\text { Flujo } \\
\text { másico de } \\
\mathbf{H}_{\mathbf{2}} \\
\text { (l.min }^{-1} \text { ) }\end{array}$ & $\begin{array}{c}\text { Flujo } \\
\text { másico de } \\
\text { Ar } \\
\text { (l.min }^{-1} \text { ) }\end{array}$ \\
\hline M1 & $\begin{array}{l}\text { Parámetro de } \\
\text { referencia }\end{array}$ & 530 & 10,8 & 43,2 \\
\hline M2 & $\begin{array}{l}\text { Disminución de } \\
\text { intensidad de } \\
\text { corriente de arco }\end{array}$ & 350 & 10,8 & 43,2 \\
\hline M3 & $\begin{array}{l}\text { Incremento } \\
\text { intensidad de } \\
\text { corriente de arco }\end{array}$ & 700 & 10,8 & 43,2 \\
\hline M4 & $\begin{array}{l}\text { Disminución } \\
\text { relación } \mathrm{H}_{2} / \mathrm{Ar}\end{array}$ & 530 & 4,9 & 49,1 \\
\hline M5 & $\begin{array}{l}\text { Incremento } \\
\text { relación } \mathrm{H}_{2} / \mathrm{Ar}\end{array}$ & 530 & 13,1 & 40,6 \\
\hline M6 & $\begin{array}{l}\text { Disminución del } \\
\text { flujo de } \mathrm{H}_{2}+\mathrm{Ar}\end{array}$ & 530 & 8,0 & 32,0 \\
\hline M7 & $\begin{array}{l}\text { Incremento del } \\
\text { flujo de } \mathrm{H}_{2}+\mathrm{Ar}\end{array}$ & 530 & 14,0 & 56,0 \\
\hline
\end{tabular}

a)

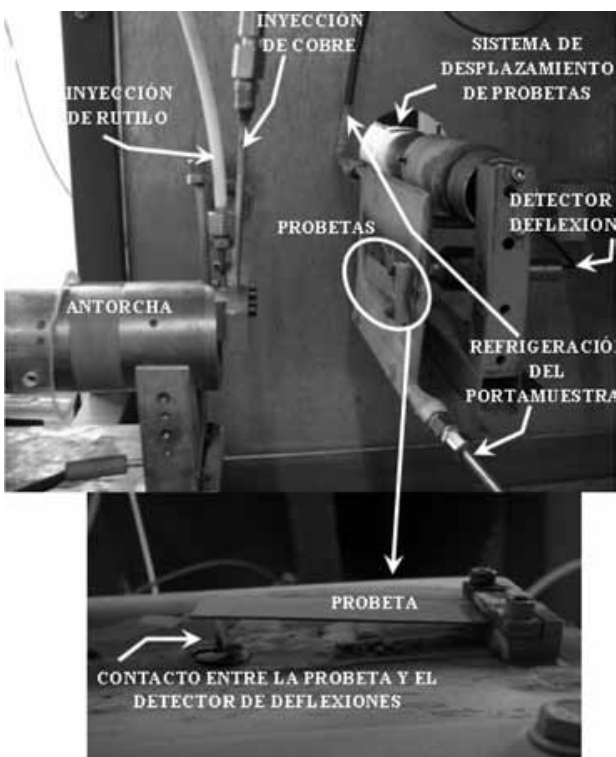

Figura 2. Sistema usado para la medición de esfuerzos residuales durante la elaboración de recubrimientos de $\mathrm{TiO}_{2}-\mathrm{Cu}$ : a) Dispositivo de proyección y portamuestras; b) Montaje de la probeta a recubrir.

$$
\sigma=\frac{\operatorname{Ed}_{\mathrm{s}}^{3}}{6(1-v) \operatorname{Rd}_{\mathrm{c}}^{2}\left(1+\frac{\mathrm{d}_{\mathrm{S}}}{\mathrm{d}_{\mathrm{c}}}\right)}
$$

Donde $\sigma$ representa el esfuerzo residual (de tensión o compresión) que estaría presente en la probeta si se impidiera su deflexión, la cual está representada en el radio de curvatura R; E corresponde al módulo de Young del material del substrato que para el caso del acero es de $210 \mathrm{GPa}, \nu$ es la relación de Poisson del material del substrato que para el acero es de 0,$3 ; d_{s}$ es el espesor del substrato y $d_{c}$ el espesor del recubrimiento aplicado.

La ecuación (1) toma en cuenta la relación substratorecubrimiento y considera que la probeta se encuentra completamente plana antes de la proyección.

Una vez calculados los esfuerzos residuales producidos en los recubrimientos, se determinó la magnitud de la deformación que dichos esfuerzos pueden producir, utilizando la ley de elasticidad de Hooke, con el fin de emplear este valor como parámetro en los cálculos realizados por el método de elementos finitos.

\section{Análisis de imágenes de los recubrimientos de $\mathrm{Al}_{2} \mathbf{O}_{\mathbf{3}}-\mathbf{1 3 T i O}_{\mathbf{2}}$}

Con el fin de obtener los dominios para posterior discretización y análisis por el Método de Elementos Finitos (MEF), se realizó observación por MEB de los recubrimientos de alúmina-óxido de titanio. Para lograr una identificación confiable de cada uno de los constituyentes de su microestructura (presencia de partículas de $\mathrm{Al}_{2} \mathrm{O}_{3}$ y $\mathrm{TiO}_{2}$ y red de poros, vacíos y grietas), la observación se realizó bajo el modo de Electrones Retro Dispersados (ERD), lo cual incrementa el contraste entre constituyentes y facilita la posterior labor de discretización del dominio del problema. La resolución de las imágenes logradas fue de 0,22 $\mu \mathrm{m}$ por píxel, ver Figura 3a.

La determinación del contenido de vacíos y la densidad de grietas en los recubrimientos de alúmina-óxido de titanio se realizó mediante el análisis de las imágenes logradas por MEB usando el programa "SCION Image" [11]. Este programa permite identificar las diferentes características de los recubrimientos y convertirlas en un sistema 
de imagen binaria, lo que facilita la posterior cuantificación de constituyentes, ver Figura $3 b$.

a)

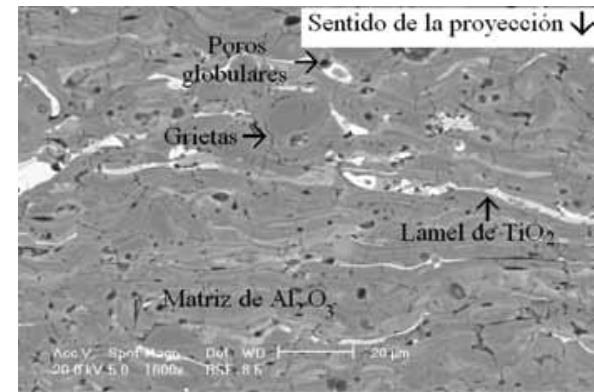

b)

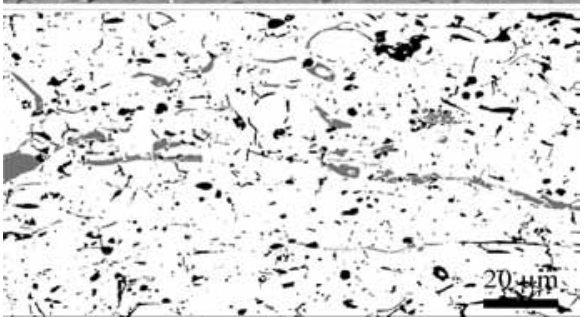

Figura 3. Microestructura de un recubrimiento de $\mathrm{Al}_{2} \mathrm{O}_{3}-13 \mathrm{TiO}_{2}$ : a) Imagen tomada mediante MEB; b) Imagen binaria después del tratamiento de la imagen original.

\section{Protocolo numérico a seguir con las imágenes de los recubrimientos de $\mathrm{Al}_{2} \mathrm{O}_{3}-\mathbf{1 3 T i O}_{2}$}

Las imágenes binarias de los recubrimientos de alúmina-óxido de titanio fueron analizadas mediante MEF luego de dividir en pequeños elementos cada uno de los constituyentes de la microestructura (matriz, constituyentes secundarios, poros y grietas), como se muestra en la Figura 4. Dicha división se realizó usando el software de uso libre PPM2OOF [10], el cual permitió enmallar el dominio con elementos triangulares formados en cada píxel de la imagen, logrando 689.210 nodos por imagen.

Para realizar los cálculos mediante MEF se tuvieron en cuenta las siguientes consideraciones: i) el modelo presenta comportamiento netamente elástico, dado que los recubrimientos estudiados son de naturaleza cerámica y su comportamiento plástico es limitado; ii) asignación de desplazamientos de igual magnitud en cada una de las dos direcciones de la imagen, ver Figura 5; iii) el módulo de Young y el coeficiente de Poisson de cada uno de los constituyentes fueron: para el $\mathrm{Al}_{2} \mathrm{O}_{3} \mathrm{E}_{\mathrm{Al} 2 \mathrm{O} 3}=370 \mathrm{GPa} \mathrm{y}_{\mathrm{Al} 2 \mathrm{O} 3}=0,22 \mathrm{y}$

para el $\mathrm{TiO}_{2} \mathrm{E}_{\mathrm{TiO} 2}=228 \mathrm{GPa}$ y $v_{\mathrm{TiO} 2}=0,27$; iv) el modelo es bidimensional; v) no se tuvo en cuenta la ocurrencia de fenómenos de deslizamiento entre las láminas que conforman el recubrimiento; vi) se asumió contacto perfecto entre las láminas, dado el hecho de que durante la elaboración de recubrimientos de alúmina-óxido de titanio proyectados a través de un chorro de plasma, se forma una capa muy delgada de $\mathrm{Al}_{\mathrm{x}} \mathrm{Ti}_{\mathrm{y}} \mathrm{O}_{5}$ que mejora la cohesión entre dichas láminas; vii) los cálculos fueron realizados bajo condiciones isotérmicas y las propiedades intrínsecas de cada uno de los constituyentes del recubrimiento se consideraron a temperatura ambiente.

Los cálculos se desarrollaron en cinco dominios discretizados (imágenes de MEB) seleccionados aleatoriamente a lo largo del recubrimiento y sus resultados se promediaron.

a)

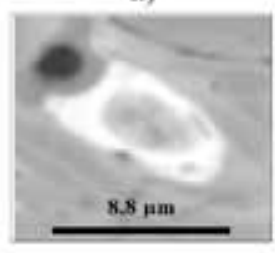

c)

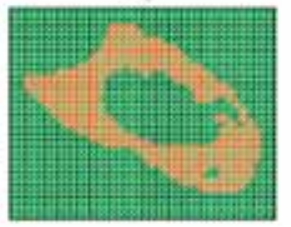

c)

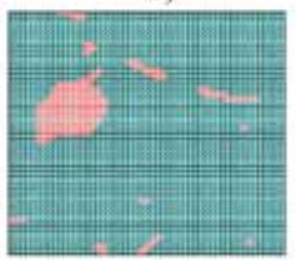

Figura 4. Proceso de discretización en cada constituyente del recubrimiento de $\mathrm{Al}_{2} \mathrm{O}_{3}$ - $\mathrm{TiO}_{2}$ : a) Imagen de MEB en escala de grises; b) imagen binaria; c) malla en el $\mathrm{TiO}_{2}$; d) malla en la matriz de $\mathrm{Al}_{2} \mathrm{O}_{3}$; e) malla en los poros y la red de grietas. 
a)

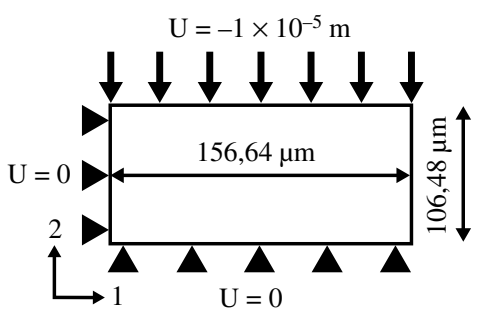

b)

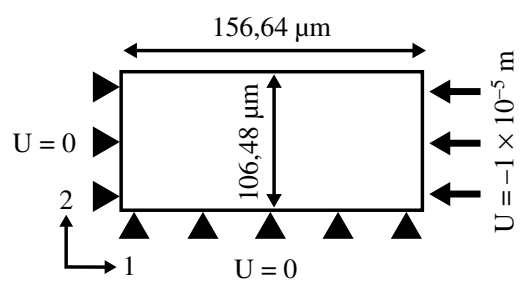

Figura 5. Desplazamientos de compresión aplicados al análisis por MEF de las imágenes de los recubrimientos de $\mathrm{Al}_{2} \mathrm{O}_{3}-13 \mathrm{TiO}_{2}$ : a) carga compresiva transversal (perpendicular al substrato); b) carga compresiva longitudinal (paralela al substrato).

La cantidad de imágenes fue deducida de un análisis convencional de la variabilidad, que consistió en: i) realizar el análisis mediante $\mathrm{MEF}$ de 15 imágenes correspondientes a un mismo recubrimiento; ii) determinar los valores del máximo esfuerzo; iii) promediarlos para diferente cantidad de imágenes; y iv) calcular sus respectivas desviaciones estándar. Posteriormente, se graficó la relación de variabilidad (desviación estándar / promedio del esfuerzo) para las distintas cantidades de imágenes, como se presenta en la Figura 6, en la cual se observa que la relación de variabilidad tiende a valores constantes a partir de cinco imágenes, significando esto que los resultados que se obtengan con mayor cantidad de imágenes arrojarán valores muy similares, por lo tanto, analizar cinco imágenes resulta suficiente.

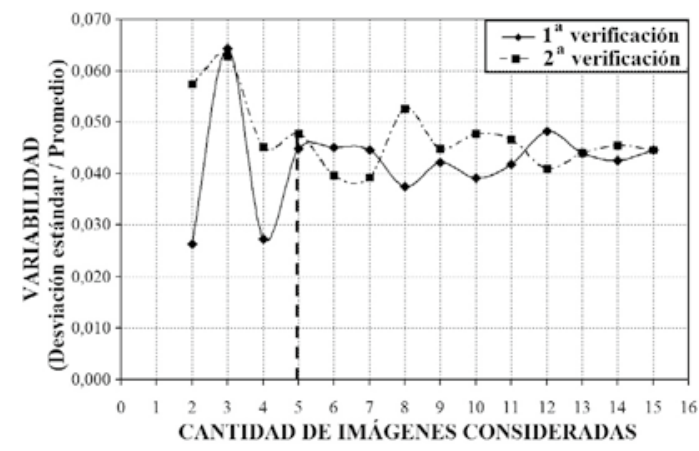

Figura 6. Variabilidad en función de la cantidad de imágenes tratadas de los recubrimientos de $\mathrm{Al}_{2} \mathrm{O}_{3}-13 \mathrm{TiO}_{2}$.

Adicionalmente, el modelo numérico fue complementado con la determinación del módulo de Young aparente de los recubrimientos considerando desplazamientos negativos en la dirección perpendicular y paralela al substrato, como se muestra en las figuras 5 a y 5 b, respectivamente y cuya magnitud fue de $1 \times 10^{-5} \mathrm{~m}$.

La secuencia de procedimientos y análisis seguidos para la obtención de resultados se resume en la Figura 7.

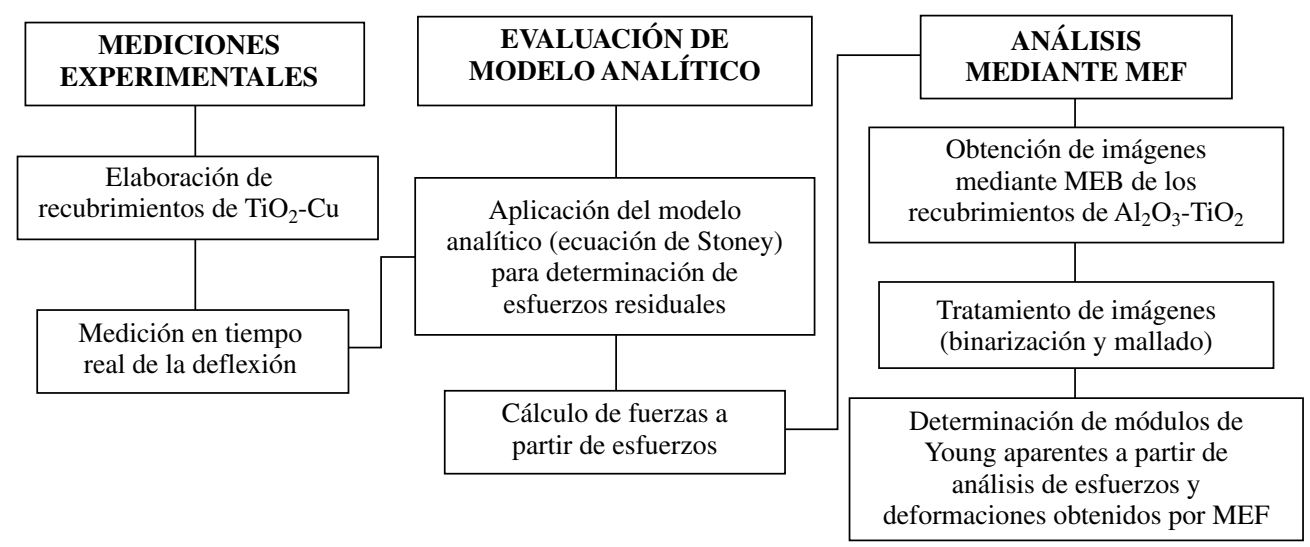

Figura 7. Diagrama de flujo de procedimientos y análisis realizados para la obtención de los resultados. 


\section{RESULTADOS}

\section{Esfuerzos residuales en los recubrimientos de $\mathrm{TiO}_{2}-\mathrm{Cu}$}

Se registró en tiempo real la deflexión durante la proyección, desde su inicio y hasta enfriamiento total, como se muestra en la Figura 8. Posteriormente, el esfuerzo residual fue calculado mediante la ecuación de Stoney, ecuación (1).

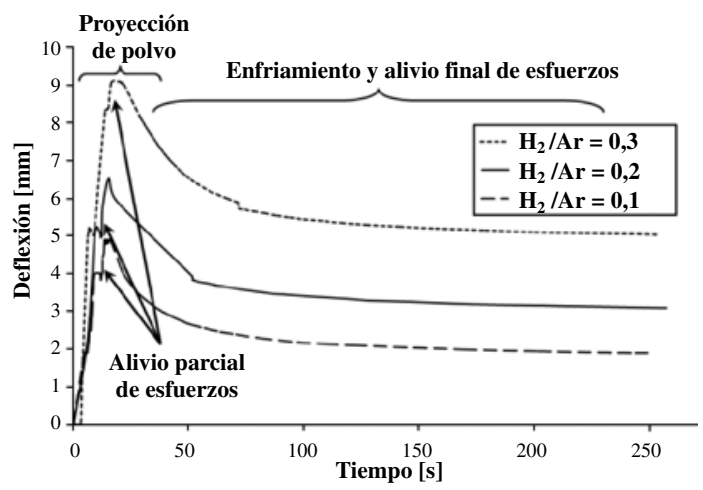

Figura 8. Comportamiento de las deflexiones para diferentes relaciones $\mathrm{H}_{2} /$ Ar.

El calor suministrado al substrato por el chorro de plasma produce una deformación negativa importante. No obstante, durante la proyección del polvo, el comportamiento de las deflexiones se ve parcialmente afectado por: i) el impacto del polvo sobre el substrato; ii) la solidificación de las partículas; iii) la transferencia de calor entre las partículas calientes y el substrato, induciendo un alivio parcial de esfuerzos mientras que el recubrimiento se va formando.

Con el fin de determinar el efecto de estos factores sobre la deflexión final, se sometieron algunos substratos únicamente a la acción del chorro de plasma producido con cada una de las mezclas de $\mathrm{H}_{2} / \mathrm{Ar}$, encontrando que este produce una deflexión contraria a la producida por el efecto de las partículas y de la formación del recubrimiento. En tal sentido, al determinar el gradiente de deflexión (deflexión final menos deflexión producida por el efecto térmico del chorro de plasma) se encontró que en las muestras elaboradas con las mezclas de $\mathrm{H}_{2} / \mathrm{Ar}$ de 0,1 y 0,2 el gradiente de deflexión es positivo y los esfuerzos residuales correspondientes, determinados mediante la ecuación (1), fueron 62 y $151 \mathrm{MPa}$, respectivamente, para sus correspondientes espesores de recubrimiento de $206 \mu \mathrm{m}$ y $200 \mu \mathrm{m}$. Esto indica que el alivio de la deflexión por el impacto de las partículas y la formación del recubrimiento es superior a la deformación causada por el calor del chorro de plasma. Por su parte, en las muestras obtenidas utilizando la mezcla de $\mathrm{H}_{2} / \mathrm{Ar}$ de 0,3 , la deflexión negativa producida por el chorro de plasma es tan alta que las deformaciones asociadas a la formación del recubrimiento no son suficientes para contrarrestarla, obteniendo un esfuerzo residual de $188 \mathrm{MPa}$ para un espesor de recubrimiento de $174 \mu \mathrm{m}$. Ver Figura 9.

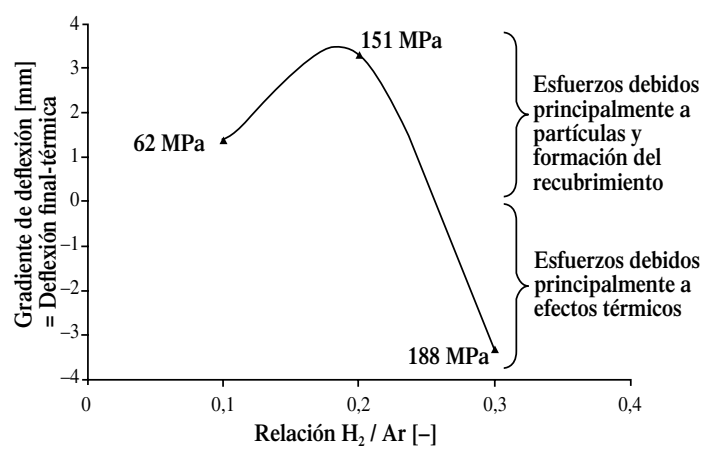

Figura 9. Comportamiento del gradiente de las deflexiones durante la elaboración de los recubrimientos de $\mathrm{TiO}_{2}-\mathrm{Cu}$ a diferentes relaciones $\mathrm{H}_{2} / \mathrm{Ar}$.

El efecto de la formación del recubrimiento sobre los esfuerzos residuales es producto de la diferencia entre los coeficientes de dilatación térmica lineal de los materiales involucrados, los cuales se muestran en la Tabla 3.

Tabla 3. Coeficientes de dilatación térmica lineal a $25^{\circ} \mathrm{C}$ para los polvos $\left(\mathrm{TiO}_{2}\right.$ y $\left.\mathrm{Cu}\right)$ y el substrato empleados [15].

\begin{tabular}{|c|c|}
\hline \multirow{2}{*}{ Material } & $\alpha \mathbf{a ~ 2 5}{ }^{\circ} \mathbf{C}$ \\
\cline { 2 - 2 } & $\left(\boldsymbol{\mu} \mathbf{m}^{-\mathbf{1}}{ }^{\circ} \mathbf{C}\right)$ \\
\hline Rutilo & 7,1 \\
\hline Cobre & 16,4 \\
\hline Acero de bajo carbono & 11,6 \\
\hline
\end{tabular}

Defectos en la estructura de los recubrimientos de $\mathrm{Al}_{2} \mathrm{O}_{3}-\mathrm{TiO}_{2}$

Los resultados del análisis de imágenes realizado a los recubrimientos de $\mathrm{Al}_{2} \mathrm{O}_{3}-\mathrm{TiO}_{2}$, con el fin de 
determinar la cantidad y distribución de defectos (poros y grietas), son mostrados en la Tabla 4 [14].

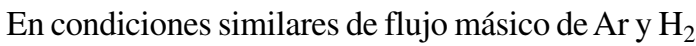
utilizados para la formación del chorro de plasma (ver condiciones de elaboración de las muestras M1, M2 y M3 en la Tabla 2), la cantidad de poros en el recubrimiento es significativamente afectada por la variación en la intensidad de corriente, como se evidencia en la Tabla 4. De hecho, dichos parámetros controlan la entalpía en el plasma (intensidad de corriente eléctrica aplicada para producir el arco, relación $\mathrm{H}_{2} /$ Ar y flujo de $\mathrm{Ar}+\mathrm{H}_{2}$, principalmente), el intercambio térmico entre el plasma y las partículas (relación $\mathrm{H}_{2} / \mathrm{Ar}$ ) y el tiempo de residencia de las partículas en el chorro de plasma.

Adicionalmente, se identificó que la relación $\mathrm{H}_{2} / \mathrm{Ar}$ (ver condiciones de proyección de las muestras M1, M4 y M5 en la Tabla 2) es la que tiene la mayor incidencia sobre la densidad de grietas (cantidad de grietas paralelas y perpendiculares a la superficie del substrato por unidad de longitud), como se puede ver en la Tabla 4. De hecho, mientras mayor es la relación $\mathrm{H}_{2} / \mathrm{Ar}$ mayor es el flujo de calor que el plasma transfiere hacia el substrato. En consecuencia, a mayor temperatura en el substrato y menor resistencia térmica en la interfaz durante la solidificación de las partículas, mayor es la velocidad de enfriamiento.

Como resultado de ello, la mayor densidad de grietas se puede asociar con una mayor velocidad de enfriamiento en cada lámina del recubrimiento. Asimismo, a mayor temperatura en el substrato se obtienen mayores esfuerzos residuales como resultado del enfriamiento, los cuales al aliviarse pueden llegar a agrietar el recubrimiento cerámico.

\section{Módulos de Young aparentes de los recubrimientos de $\mathrm{Al}_{2} \mathrm{O}_{3}-\mathrm{TiO}_{2}$}

La Tabla 5 contiene los módulos de Young aparentes de los recubrimientos elaborados bajo los diferentes parámetros de operación presentados en la Tabla 2 y siguiendo el procedimiento detallado en el Protocolo Numérico para el análisis mediante MEF.

Como ya se mencionó, las propiedades mecánicas aparentes de los recubrimientos elaborados mediante proyección térmica por plasma dependen de factores como la distribución espacial y de tamaño de los constituyentes del recubrimiento en conjunto con la arquitectura porosa. No obstante, otros factores, como la geometría y conectividad de poros y grietas en la arquitectura porosa del recubrimiento, pueden influir en los valores del módulo de Young aparente, produciendo valores de esfuerzos concentrados en el recubrimiento, lo que se refleja en valores del módulo de Young aparente mayores al mayor módulo de Young considerado en los constituyentes del recubrimiento (en este caso $370 \mathrm{GPa}$, correspondiente al $\mathrm{Al}_{2} \mathrm{O}_{3}$ ), como ocurre con las muestras M1, M5 y M6 presentadas en la Tabla 5 y como se detalla en la Tabla 6.

La Tabla 6 muestra las correlaciones entre las características estructurales de los recubrimientos y sus módulos de Young aparentes presentados en orden ascendente de los valores reportados en la Tabla 5. Se puede observar claramente que el módulo de Young aparente de los recubrimientos se ve directamente afectado por la relación entre grietas paralelas y normales al substrato normalizando al porcentaje total de poros.

Tabla 4. Contenido de poros y grietas en los recubrimientos de $\mathrm{Al}_{2} \mathrm{O}_{3}-13 \mathrm{TiO}_{2}$ elaborados con diferentes parámetros de operación [14].

\begin{tabular}{|c|c|c|c|c|c|}
\hline \multirow{2}{*}{ Grupo } & \multicolumn{3}{|c|}{ Porcentaje de defectos (\%) } & \multicolumn{2}{c|}{ Densidad de grietas $\left(\mathbf{m m}^{-\mathbf{1}}\right)$} \\
\cline { 2 - 6 } & Poros globulares & Microgrietas & Total & $\begin{array}{c}\text { Paralela } \\
\text { al substrato }\end{array}$ & $\begin{array}{c}\text { Normal } \\
\text { al substrato }\end{array}$ \\
\hline M1 & $1,5 \pm 0,1$ & $4,8 \pm 0,2$ & $6,3 \pm 0,3$ & $7,3 \pm 0,7$ & $5,6 \pm 0,5$ \\
\hline M2 & $2,1 \pm 0,1$ & $5,1 \pm 0,1$ & $7,2 \pm 0,3$ & $10,0 \pm 0,5$ & $6,2 \pm 0,4$ \\
\hline M3 & $1,2 \pm 0,1$ & $4,5 \pm 0,1$ & $5,7 \pm 0,2$ & $6,9 \pm 0,6$ & $4,0 \pm 0,3$ \\
\hline M4 & $1,8 \pm 0,2$ & $4,6 \pm 0,3$ & $6,4 \pm 0,5$ & $8,9 \pm 1,3$ & $2,9 \pm 0,4$ \\
\hline M5 & $1,9 \pm 0,2$ & $5,3 \pm 0,1$ & $7,2 \pm 0,3$ & $8,6 \pm 0,4$ & $6,2 \pm 0,4$ \\
\hline M6 & $3,3 \pm 0,3$ & $5,3 \pm 0,1$ & $8,6 \pm 0,4$ & $9,7 \pm 0,7$ & $6,7 \pm 0,4$ \\
\hline M7 & $1,1 \pm 0,1$ & $4,2 \pm 0,1$ & $5,3 \pm 0,3$ & $5,9 \pm 0,5$ & $2,8 \pm 0,3$ \\
\hline
\end{tabular}


Tabla 5. Esfuerzos y módulos de Young aparentes calculados bajo carga de compresión perpendicular al substrato en los recubrimientos de $\mathrm{Al}_{2} \mathrm{O}_{3}-13 \mathrm{TiO}_{2}$.

\begin{tabular}{|c|c|c|c|}
\hline \multicolumn{2}{|c|}{ Grupo } & Esfuerzo [MPa] & $\begin{array}{c}\text { Módulos de Young } \\
\text { aparentes (GPa) }\end{array}$ \\
\hline M1 & Parámetros de referencia & $-3,91 \pm 0,13$ & $390,5 \pm 13,4$ \\
\hline M2 & Disminución intensidad de corriente de arco & $-3,64 \pm 0,19$ & $363,9 \pm 19,1$ \\
\hline M3 & Incremento intensidad de corriente de arco & $-3,63 \pm 0,36$ & $362,6 \pm 35,8$ \\
\hline M4 & Disminución relación $\mathrm{H}_{2} / \mathrm{Ar}$ & $-3,12 \pm 0,18$ & $312,1 \pm 17,9$ \\
\hline M5 & Incremento relación $\mathrm{H}_{2} / \mathrm{Ar}$ & $-4,21 \pm 0,22$ & $421,4 \pm 21,5$ \\
\hline M6 & Disminución flujo de $\mathrm{H}_{2}+\mathrm{Ar}$ & $-4,24 \pm 0,14$ & $424,3 \pm 14,3$ \\
\hline M7 & Incremento flujo de $\mathrm{H}_{2}+\mathrm{Ar}$ & $-3,38 \pm 0,48$ & $338,6 \pm 47,8$ \\
\hline
\end{tabular}

Tabla 6. Correlación entre las características estructurales de los recubrimientos y sus módulos de Young aparentes.

\begin{tabular}{|l|c|c|c|c|c|c|c|}
\hline \multirow{2}{*}{ Relación } & \multicolumn{7}{|c|}{ Módulo de Young aparente (GPa) } \\
\cline { 2 - 9 } & $\mathbf{3 1 2 , 1}$ & $\mathbf{3 3 8 , 6}$ & $\mathbf{3 6 2 , 6}$ & $\mathbf{3 6 3 , 9}$ & $\mathbf{3 9 0 , 5}$ & $\mathbf{4 2 1 , 4}$ & $\mathbf{4 2 4 , 3}$ \\
\cline { 2 - 9 } & $\mathbf{M 4}$ & $\mathbf{M 7}$ & $\mathbf{M 3}$ & $\mathbf{M 2}$ & $\mathbf{M 1}$ & $\mathbf{M 5}$ & $\mathbf{M 6}$ \\
\hline $\begin{array}{l}\text { Densidad de grietas paralelas al substrato/ } \\
\text { porcentaje total de poros }\end{array}$ & 1,41 & 1,11 & 1,19 & 1,37 & 1,16 & 1,18 & 1,91 \\
\hline $\begin{array}{l}\text { Densidad de grietas normales al substrato/ } \\
\text { porcentaje total de poros }\end{array}$ & 0,46 & 0,53 & 0,69 & 0,85 & 0,89 & 0,85 & 0,78 \\
\hline $\begin{array}{l}\text { (Densidad de grietas paralelas al substrato/ } \\
\text { densidad de grietas normales al substrato) } \\
\text { /porcentaje total de poros }\end{array}$ & 0,49 & 0,40 & 0,30 & 0,22 & 0,21 & 0,19 & 0,17 \\
\hline $\begin{array}{l}\text { (Densidad total de grietas/contenido de } \\
\text { poros globulares)/porcentaje total de poros }\end{array}$ & 1,87 & 1,64 & 1,88 & 2,22 & 2,05 & 2,03 & 0,19 \\
\hline
\end{tabular}

Anisotropía en los recubrimientos de $\mathrm{Al}_{2} \mathrm{O}_{3}-\mathbf{1 3 T i O}_{2}$ Con el propósito de evaluar la anisotropía del recubrimiento, se aplicó al modelo un desplazamiento en dirección paralela al substrato en los recubrimientos de $\mathrm{Al}_{2} \mathrm{O}_{3}-13 \mathrm{TiO}_{2}$. Los resultados se confrontaron con los obtenidos en el modelo aplicando carga perpendicular al substrato. La Tabla 7 sintetiza los resultados obtenidos y evidencia que, en efecto, los recubrimientos exhiben un comportamiento anisotrópico, presentando módulos de Young menores cuando la carga se aplica en dirección paralela al substrato que cuando se hace en dirección perpendicular al mismo, lo cual resulta como consecuencia del contenido de grietas paralelas al substrato, como se presenta en la Tabla 4. Mayores contenidos de grietas paralelas al substrato, que de grietas perpendiculares al mismo, en conjunto con el contenido de poros, reducen el área efectiva de distribución de la carga en esta dirección, provocando
Tabla 7. Módulos de Young aparentes de los recubrimientos de $\mathrm{Al}_{2} \mathrm{O}_{3}-13 \mathrm{TiO}_{2}$ determinados bajo cargas perpendicular y paralela al substrato.

\begin{tabular}{|c|c|c|}
\hline \multirow{2}{*}{$\begin{array}{c}\text { Zona del } \\
\text { recubrimiento }\end{array}$} & \multicolumn{2}{|c|}{$\begin{array}{c}\text { Módulo de Young } \\
\text { aparente (GPa) }\end{array}$} \\
\cline { 2 - 3 } & $\begin{array}{c}\text { Bajo carga } \\
\text { I * }\end{array}$ & $\begin{array}{c}\text { Bajo carga } \\
\text { II** }\end{array}$ \\
\hline A & 373,6 & 270,7 \\
\hline B & 392,2 & 295,2 \\
\hline C & 402,9 & 281,5 \\
\hline D & 403,5 & 292,5 \\
\hline E & 380,1 & 268,5 \\
\hline Valor promedio & 390,5 & 281,7 \\
\hline $\begin{array}{c}\text { Desviación } \\
\text { estándar }\end{array}$ & 13,4 & 12,2 \\
\hline
\end{tabular}

* Carga con orientación perpendicular al substrato.

** Carga con orientación paralela al substrato. 
mayores esfuerzos al interior del recubrimiento y en consecuencia exhibiendo módulos de Young mayores.

Igualmente, bajo condiciones de carga paralela al substrato, se notó que el módulo de Young aparente está afectado de manera importante por el contenido de los constituyentes secundarios $\left(\mathrm{TiO}_{2}\right)$ y la arquitectura porosa, como lo explican los modelos de Reuss (singularidades en serie) y de Voigt (singularidades en paralelo) [17], los cuales se ilustran en la Tabla 8.

Por otra parte, se puede señalar que hay una muy buena transferencia de esfuerzo de la matriz a las partículas del segundo constituyente (por la cohesión perfecta considerada en el modelo). Por el contrario, los vacíos y grietas en el recubrimiento representan discontinuidades en la estructura y, por lo tanto, facilitan el desarrollo de gradientes de esfuerzo alrededor de dichas discontinuidades, como se muestra en la Figura 10. En consecuencia, se incrementa la sensibilidad al daño localizado en las zonas adyacentes a las discontinuidades presentes en el recubrimiento.

Tabla 8. Modelos en serie y en paralelo para materiales compuestos [17].

\begin{tabular}{|c|}
\hline Modelo de Reuss (modelo en serie) \\
\hline \\
\hline
\end{tabular}

a)

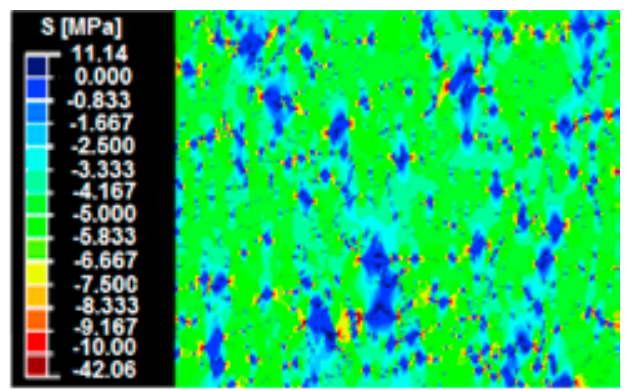

b)

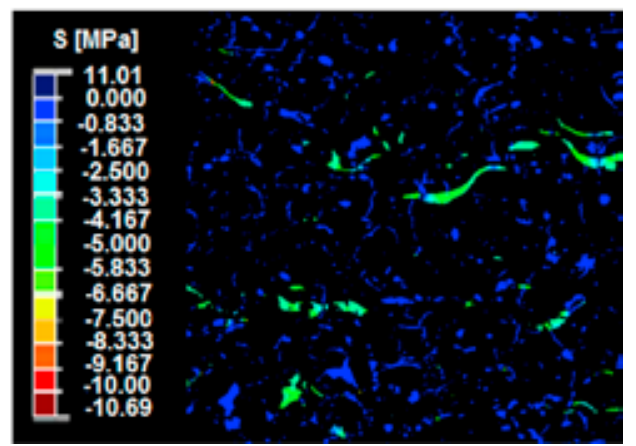

Figura 10. Distribución de esfuerzos en los recubrimientos de $\mathrm{Al}_{2} \mathrm{O}_{3}-\mathrm{TiO}_{2}$ elaborados por proyección térmica por plasma: a) Distribución de esfuerzos en la matriz de $\mathrm{Al}_{2} \mathrm{O}_{3} ;$ b) Distribución de esfuerzos en los poros, grietas y partículas de segunda fase $\left(\mathrm{TiO}_{2}\right)$.

El modelo de Kachanov [18] define el Parámetro de Daño D, en función de la superficie afectada por las discontinuidades, así:

$$
\mathrm{D}=\frac{\mathrm{S}^{*}}{\mathrm{~S}} \quad \operatorname{con} 0<\mathrm{D}<1
$$

donde $\mathrm{S}$ es la superficie libre de poros o grietas y $\mathrm{S}^{*}$ es la superficie ocupada por los poros y grietas.

De acuerdo con la ecuación (2), la superficie efectiva sobre la cual el esfuerzo se distribuye afecta al módulo de Young disminuyendo su valor a medida que aumenta $\mathrm{D}$, según la ecuación (3):

$$
\mathrm{E}_{\mathrm{a}}=\mathrm{E}(1-\mathrm{D})
$$

donde $\mathrm{E}_{\mathrm{a}}$ es el módulo de Young afectado por el parámetro de daño D y E corresponde al módulo de Young del material ideal, es decir, libre de defectos o discontinuidades. 
a)

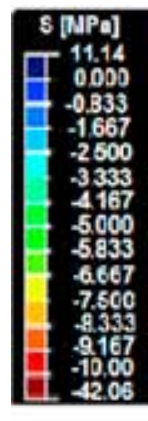

b)

c)

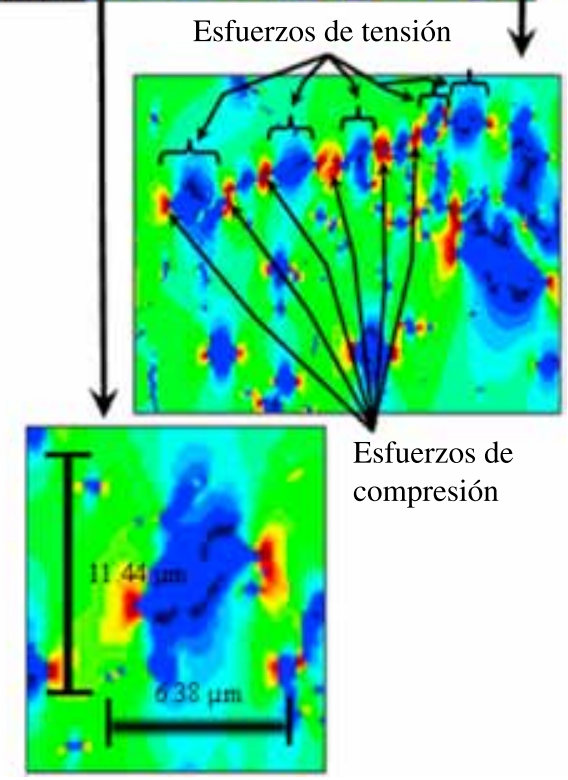

Figura 11. Distribución microestructural de esfuerzos:

a) Distribución de esfuerzos en la matriz de $\mathrm{Al}_{2} \mathrm{O}_{3}$; b) Esfuerzos de tensión y compresión; c) Ejemplo de los gradientes de esfuerzo en la matriz.

Asimismo, es importante señalar que la interacción entre la matriz y la arquitectura porosa varía entre esfuerzos de tensión y de compresión, como se muestra en la Figura 11. Los esfuerzos de mayor magnitud se concentran en la matriz cerca a los poros y grietas, siendo del orden de 11,14 MPa, como lo muestra la Figura 11c. Además, a medida que los poros son más pequeños, mayor es el gradiente de esfuerzo, favoreciendo el desarrollo y propagación de grietas en estas zonas. Esto significa que las zonas del recubrimiento que posean discontinuidades agudas son más propensas a concentrar esfuerzos y por lo tanto a facilitar la ocurrencia de falla.

\section{Comportamiento de diferentes recubrimientos teóricos}

Con el fin de estudiar la influencia de las propiedades mecánicas intrínsecas de los constituyentes sobre las propiedades mecánicas aparentes del recubrimiento (a pesar de la arquitectura porosa presente), las propiedades correspondientes $\mathrm{a}_{2} \mathrm{O}_{3}$ y TiO$_{2}$ fueron intercambiadas en el modelo de MEF (la matriz se consideró como de $\mathrm{TiO}_{2}$ y las partículas de segunda fase de $\mathrm{Al}_{2} \mathrm{O}_{3}$ ). Los cálculos se desarrollaron bajo condiciones de carga transversal, encontrando que el módulo de Young aparente del recubrimiento está principalmente influenciado por el módulo de Young intrínseco de la matriz, como lo muestra la Figura 12.

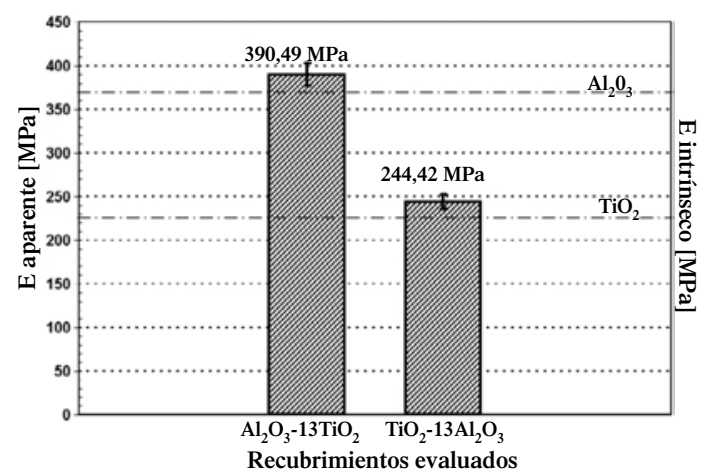

Figura 12. Influencia de las propiedades mecánicas intrínsecas de los constituyentes del recubrimiento sobre sus propiedades mecánicas aparentes.

\section{CONCLUSIONES}

En este estudio se desarrolló una metodología para el análisis mecánico de recubrimientos elaborados mediante proyección térmica por plasma, que muestra ventajas, como la posibilidad de realizar mediciones experimentales para la determinación de los esfuerzos residuales, así como la posibilidad de realizar análisis mediante MEF con modelos geométricos obtenidos a partir de microestructuras reales. Las mediciones de deflexiones permitieron identificar el origen de los esfuerzos residuales que se generan durante la elaboración del recubrimiento. Por otra parte, el análisis mediante MEF resulta flexible dado que permite seleccionar el tipo de esfuerzo mecánico a aplicar en el modelo (estacionario, transiente, etc.), facilitando la interpretación del 
comportamiento mecánico de los recubrimientos desde un punto de vista global (en la escala del recubrimiento), tanto como a nivel local (en la escala de las láminas o poros). Esta metodología puede ser útil en la definición de parámetros de elaboración de recubrimientos con miras a maximizar sus propiedades mecánicas. Por supuesto, la metodología representa una aproximación simplificada, lo cual permite identificar ciertas desventajas:

i) el análisis que se realiza mediante MEF se basa en modelos bidimensionales (los efectos tridimensionales no son tomados en cuenta);

ii) los modelos aplicados son puramente elásticos y en consecuencia los fenómenos de deslizamiento entre láminas no son considerados (ello puede modificar considerablemente el desempeño mecánico del recubrimiento);

iii) las discontinuidades de tamaños inferiores a la resolución de las imágenes no fueron consideradas en los análisis pese a que ellas pueden jugar un rol importante, en particular si se trata de grietas.

Esto explica por qué los módulos de Young aparentes calculados se ven sobrevalorados.

El módulo de Young aparente de los recubrimientos elaborados mediante proyección térmica por plasma se ve afectado por la proporción de grietas paralelas y perpendiculares al substrato y por el contenido de vacíos presentes en la microestructura, como se evidenció en los Resultados. Esto demuestra claramente una sinergia entre la red de defectos y el comportamiento elástico del recubrimiento. La optimización de los parámetros de proyección permite controlar las características de la estructura del recubrimiento y, por lo tanto, sus propiedades elásticas.

Se observó una buena transferencia de esfuerzo entre la matriz de alúmina y la segunda fase de óxido de titanio embebida en ella, pese a los gradientes de esfuerzo que se desarrollan en los alrededores de la red de poros. Estas discontinuidades incrementan la sensibilidad al daño localizado del recubrimiento en las zonas adyacentes y posibilitan el desarrollo de grietas que pueden conducir a falla en el recubrimiento. Adicionalmente, el módulo de Young aparente del recubrimiento compuesto es principalmente influenciado por el módulo de Young intrínseco de la matriz. Modelos simples como los de Voigt, Reuss y Kachanov fueron muy útiles para interpretar estos comportamientos.

\section{AGRADECIMIENTOS}

El autor del presente artículo agradece a los doctores Guy Antou, Alain Denoirjean, Alain Grimaud y Ghislain Montavon del laboratorio SPCTS de la Facultad de Ciencias de la Universidad de Limoges, Francia, por su valiosa colaboración y aportes en el desarrollo del estudio relativo a los resultados presentados en este trabajo.

\section{REFERENCIAS}

[1] P. Araujo, D. Chicot, M. Staia and J. Lesage. "Residual Stresses and Adhesion of Thermal Spray Coatings". Surface Engineering. Vol. 21, Issue 1, pp. 35-40. February, 2005. ISSN: 0267-0844. DOI: 10.1179/174329405X30020.

[2] C.C. Berndt and P.J. Callus. "Relationships between the Mode II Fracture Toughness and Microstructure of Thermal Spray Coatings". Surface and Coatings Technologie. Vol. 114, Issue 2-3, pp. 114-128. May, 1999. ISSN: 0257-8972. DOI: $10.1016 /$ S0257-8972(99)00018-3.

[3] I. Chaieb. "Analysis and Simulation of Residual Stresses Induced by Mechanical Pre-Treatment Like Shot Peening and Laser Hardening". Ph.D. thesis. University of Reims, France. 2004.

[4] R. Gafouri-Azar, J. Mostaghimi and S. Chandra. "Modeling Development of Residual Stresses in Thermal Spray Coatings". Computational Materials Science. Vol. 35, Issue 1, pp. 13 - 26. January, 2006. ISSN: 0927-0256. DOI:10.1016/j. commatsci.2005.02.007.

[5] R. Lakes. "Extreme Damping in Compliant Composites with a Negative Stiffness Phase". Philosophical Magazine Letters. Vol. 81, Issue 2, pp. 95-100. 2001. ISSN 0950-0839. DOI: $10.1080 / 09500830010015332$.

[6] J. Matejicek and S. Sampath. "Intrinsic Residual Stresses in Single Splats Produced by Thermal Spray Processes". Acta Materialia. Vol. 49, Issue 11, pp. 1993-1999. June, 2001. ISSN: 1359-6454. DOI: $10.1016 /$ S1359-6454(01)00099-4. 
[7] S.H. Leigh and C.C. Berndt. "Modeling of Elastic Constants of Plasma Spray Deposits with Ellipsoid-Shaped Voids". Acta Materialia. Vol. 47, Issue 5, pp. 15751586. March, 1999. ISSN: 1359-6454. DOI: 10.1016/S1359-6454(99)00019-1.

[8] Z. Gan, H.W. Ng and A. Devasenapathi. "Deposition-Induced Residual Stresses in Plasma-Sprayed Coatings". Surface and Coatings Technology. Vol. 187, Issue 2-3, pp. 307-319. October, 2004. ISSN: 02578972. DOI: 10.1016/j.surfcoat.2004.02.010

[9] Dassault Systèmes. "SIMULIA > Products > Abaqus FEA". Date of renovation: 2011. Date of visit: April 20, 2011.URL: www. simulia.com/products/ abaqus_fea.html

[10] National Institute of Standards and Technology NIST. "OOF: Finite Element Analysis of Microstructures". Date of renovation: February 17, 2011. Date of visit: April 20, 2011. URL: www.ctems.nist.gov/oof/.

[11] Meyer Instruments, Inc. and Scion Corporation. "Scion Image for Windows". Date of renovation: April 26, 2000. Date of visit: April 9, 2011. URL: www.meyerinst. $\mathrm{com} / \mathrm{html} / \mathrm{scion} / \mathrm{scion} \_$image_windows.htm

[12] Electronics Incorporated. "Types and Grades of Electronics Inc. ALMEN strips”. Date of renovation: 2011. Date of visit: August 31, 2011. URL: www.electronics-inc.com/ typegrade.html.
[13] SAE International. "Standard SAE J442 Test strip, holder and gage for shot-peening". 1979.

[14] A. Coupée. "Characterization of Porous Network of Thermal Spray Coatings by Electrochemical Impedance Spectroscopy and Image Analysis". Institute National des Sciences Appliquées de Strasbourg, Laboratoire d'Ingénierie des Surfaces, pp. 4-6. 2004.

[15] O. Kraft and H. Gao. "Diffusion processes in advanced technological materials, Measurement of residual stresses in thin films and their relaxation". Devendra Gupta, IBM, Thomas J. Watson Research Center. Yorktown, NY, USA, pp. 365-404. 2005. ISBN: 978-0-8155-1501-2.

[16] MatWeb LLC. "Online Materials Information Resource - Matweb". Date of visit: April 20, 2011. URL: www.matweb.com

[17] B.M. Caruta. "New Developments in Materials Science Research". Nova Science Publishers, Inc. Nueva York, Estados Unidos. pp. 99. 2007. ISBN: 1-59454-854-4.

[18] J. Chaboche. "Damage Mechanics, Comprehensive Structural Integrity". Elsevier. Lausanne, Swizerland, pp. 215219. 2003. ISBN 10: 0-08-043749-4, ISBN 13: 978-0-08-043749-1. URL: www. elsevier.com/wps/find/bookdescription. cws_home/718179/description\#description 